\title{
Realization of a scalable airborne radar
}

\author{
D. van Halsema ${ }^{\# 1}$, R.V. de Jongh ${ }^{* 2}$, J. van Es ${ }^{* * 3}$, \\ M.P.G. Otten ${ }^{\# 4}$, B.C.B. Vermeulen ${ }^{\# 5}$, L.J. van Liempt ${ }^{* * 6}$ \\ \# TNO Defence Security and Safety \\ PO Box 96864, 2509 JG The Hague, The Netherlands \\ erik.vanhalsema@tno.nl \\ matern.otten@tno.nl \\ ${ }^{5}$ ben.vermeulen@tno.nl \\ *THALES Nederland B.V. \\ PO Box 42, 7550 GD Hengelo, The Netherlands \\ ${ }^{2}$ rene.dejongh@nl.thalesgroup.com \\ ${ }^{* *}$ National Aerospace Laboratory NLR \\ PO Box 9050, 1006 BM Amsterdam, The Netherlands \\ 3jvanes@nlr.nl \\ ${ }^{6}$ liempt@nlr.nl
}

\begin{abstract}
Modern airborne ground surveillance radar systems are increasingly based on Active Electronically Scanned Array (AESA) antennas. Efficient use of array technology and the need for radar solutions for various airborne platforms, manned and unmanned, leads to the design of scalable radar systems. The design and realisation of such a system is described. At the core of the design is a new generation of Transmit-Receive modules, developed and used also for naval radar systems. The radar architecture is largely based on the MiniSAR system, which is close to completion. Design aspects are discussed for various envisaged versions of the scalable radar.
\end{abstract}

\section{INTRODUCTION}

In the Netherlands, the development of a small radar system, MiniSAR [1], is nearing completion. MiniSAR is designed to be compatible with small unmanned aircraft such as the Sperwer in service with the Royal Netherlands Army. This design is also the basis for extended radar versions for other small and medium size airborne platforms. These radars are intended to be mounted on different platforms and a survey of platforms potentially available in the Netherlands (military, coast guard, police, etc) was done in order to investigate possibilities for mounting and integration, and the design has focused on a pod mounted radar, which can be fitted as an autonomous sensor on several envisaged platforms, both fixed wing and helicopters, with minimum impact on the platform itself. This project is carried out under the name ART, Airborne Radar Technology.

Applications of the ART radar range from well-known military ground surveillance applications to Law Enforcement and Coast Guard tasks, disaster monitoring and environmental monitoring. To span this range of applications, compatibility with a wide range of aircraft is essential. The associatad foreseen flight envelopes are depicted in the following figure, and shows the flight envelopes on which the radar design is based. These flight envelopes have a profound impact on the cooling concept, as explained in section III.

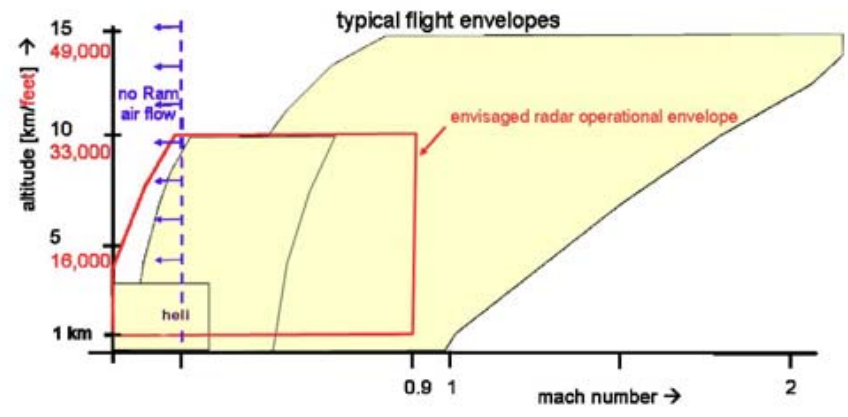

Figure 1 Foreeen typical flight envelopes

\section{SCALABLE RADAR}

The design of MiniSAR was aimed at scalability and flexibility from the start. MiniSAR itself contains 24 TR modules, operating in X-band, and the architecture and control is designed to allow extension of the number of TR modules and the number of receive channels. It is capable of SAR strip, SAR Spot and Moving Target Indication modes. For MTI operation, three receive channels are included in the design (one currently implemented).

Based on this design, with limited adjustments, the ART radar is designed in three basic versions, consisting of $24 \times 4$

\footnotetext{
${ }^{1}$ The radar system will be denoted by product name VALKYR
} 
TR modules, 48x4 modules, and 48x8 modules respectively. Obviously, the small version could be fitted to quite small platforms and the larger versions to medium and to large platforms, covering low-flying short range applications to high-flying medium range applications. The size of the array antenna varies from $40 \mathrm{~cm} \times 6 \mathrm{~cm}$, to $80 \mathrm{~cm} \times 12 \mathrm{~cm}$.

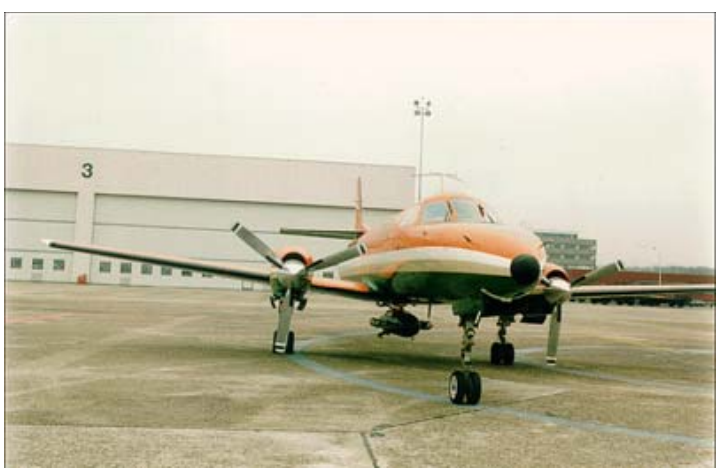

Figure 2 Example platform

\section{AIRCRAFT INTEGRATION}

\section{A. Pod design}

For ease of integration, taking into account the various platforms, the radar will be installed in a stand-alone pod, which, depending on the platform, can be mounted under the wing or the centre of the fuselage, making use of existing hard points and pylons. For helicopters also some solutions for side-mounting on existing hard points or other possibilities like existing winch constructions were identified

The pod will contain the complete radar sensor, IMU/GSP unit, data link unit, data storage unit, and on-board real-time processor. Interfaces to the platform are schematically depicted in figure 3

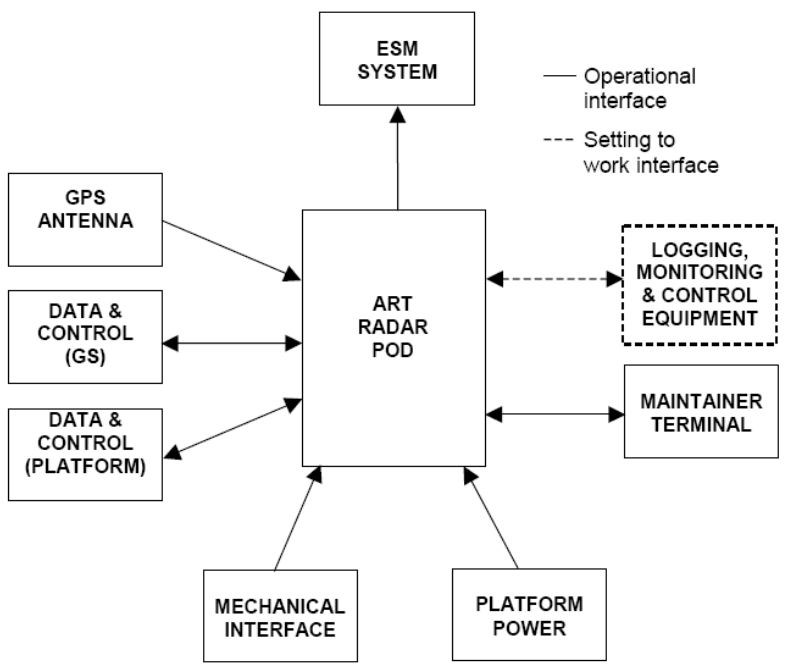

Figure 3 Interfaces between the radar pod and the aircraft

The number of connections to the platform is limited to three: power, control, and GPS antenna.

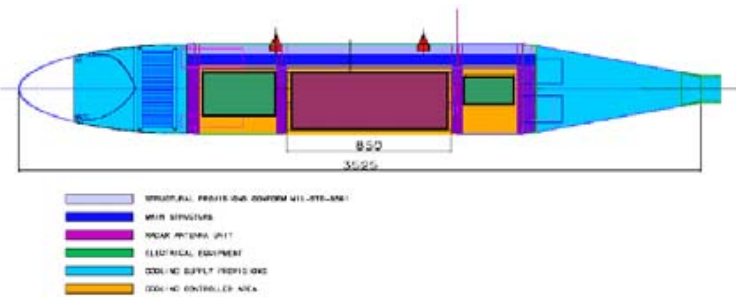

Figure 4 Schematic drawing of pod design

Inside the pod, the radar sensor, i.e. antenna and radar electronics, as well as the processor will be contained in a single rack, slightly tilted downwards $\left(15^{\circ}\right)$ to achieve an optimum side-looking configuration.

Specific integration issues such as potential multipath reflection from the wing are under investigation. Results so far have indicated that some intensity effects on the SAR image can be expected with certain platforms, which could be minimized by careful beam shape control.

\section{B. Cooling}

Cooling is an important issue, as the foreseen cooling system has to cope with potentially very different flight conditions and therefore different thermal conditions. For instance, the radar could operate on a slow and low flying platform, where outside temperature may be high and air flow limited, or on a fast flying platform with high air flows and cold air but temperature increase by air stagnation and friction. For low speed options a HX with fan design is foreseen, for high speed options an air-scoop is designed. To provide an uniform temperature in the Radar front-end a liquid pumped cooling system is used transporting the heat from the electronics to the air heat exchangers.

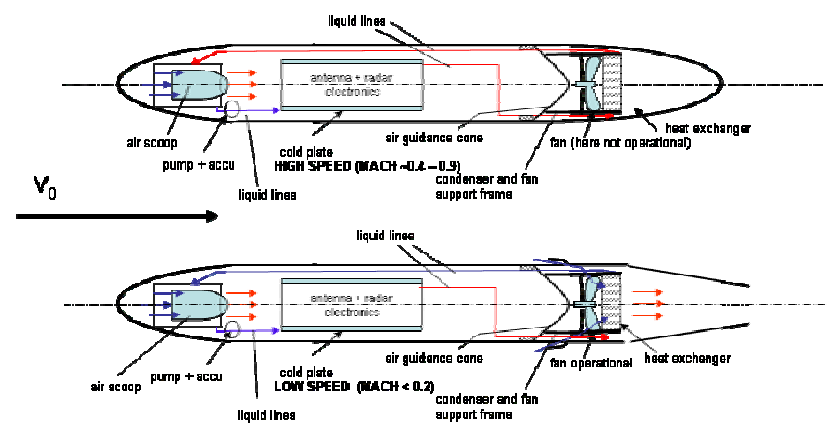

Figure 5 Pod cooling system concept 


\section{RADAR ARCHITECTURE}

The radar architecture is schematically depicted in the figure 6 , showing the complete system decomposition.

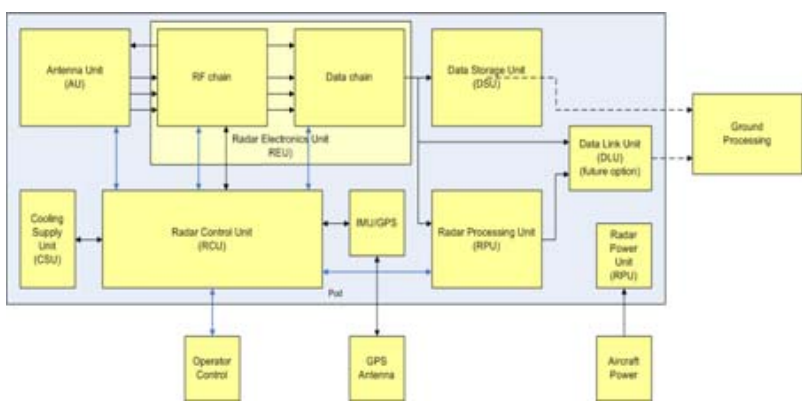

Figure 6 System decomposition

Furthermore, the entire unit is mounted in a two-rack solution as shown in figure 7. The lower slots contain the TR modules directly connected to the radiating panel, while the upper slots contain the radar electronics, RF and digital, radar control, IMU/GSP and radar processor.

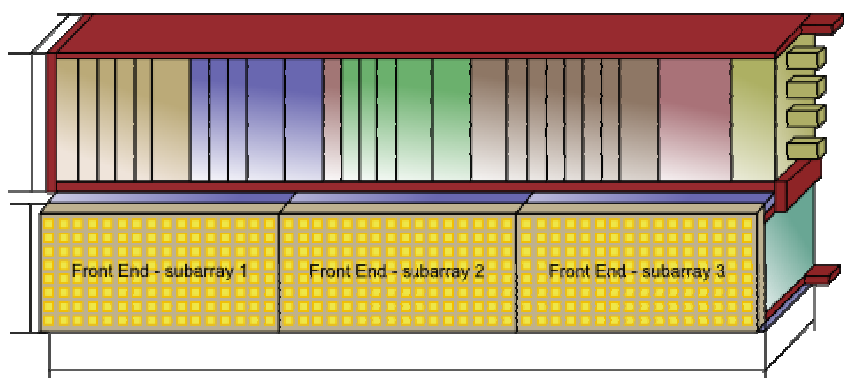

Figure 7 Complete rack-mounted radar system

The depicted version contains $48 \times 8$ (or 4) TR modules.

The radar features fully flexible (DDS) waveform generation and pulse-to-pulse setting of waveform and antenna parameters, so that complex radar modes can be realised. Moreover, the three sub-panels and receive channels allow advanced Moving Target Indication using Space-Time Adaptive Processing (STAP).

The RF and Data chain is described in the following: Radar Signal Generator generates FM chirps with a programmable bandwidth, starting with a Direct Digital Chirp Synthesizer (DDCS) for maximum waveform flexibility, generating a chirp at $1750 \mathrm{MHz}$, and with up to $500 \mathrm{MHz}$ bandwidth. The Local Oscillator Frequencies module generates the appropriate clocks and LO signals from a $125 \mathrm{MHz}$ reference signal from the Reference Generator, using only frequency multipliers and mixers (no PLLs) for optimum phase noise performance and inherent coherency over pulses. The IF/LO/RF module converts the signals to/ from the microwave frequency domain used in the Antenna Unit, and forwards the received signals to the Receiver, which can select either three subarray signals for
MTI operation, or a combined signal for normal SAR operation. The Receiver performs the final frequency downconversion to $250 \mathrm{MHz}$ IF and has variable gain for optimum matching of the radar RF Chain to the ADC characteristics of the Data Chain.

The high speed Analogue to Digital Converter (ADC) of the Data Chain converts the analogue input to digital values at the full acquisition speed of $1 \mathrm{GS} / \mathrm{s}$, converted to a wider but slower data rate by the de-multiplexer, and buffered in the FIFO to spread the high peak data rate to a lower average data rate. The timing and synchronisation functionality that ensures that tight (sub-ns) synchronisation is ensured between signal generation in the Radar Signal Generator of the RF Chain and the signal digitisation in the Data Chain. All timing falls on a common $25 \mathrm{MHz}$ grid controlled by the Radar Control Unit, which in turn derives it from the $125 \mathrm{MHz}$ Reference Generator signal; the tight coupling of clocks also ensures inherent coherency across pulses in interleaved pulse modes.

A "pre-processing" block performs coherent I/Q demodulation to a complex base band signal, filtered in the interpolating decimating FIR filter that allows high accuracy for both wide-band SARmode and narrow-band MTI-mode. After filtering, presuming (or azimuth filtering) is performed, which sums several range pulses to increase signal to noise ratio and limits the bandwidth and data-rate of the azimuth spectrum. Provisions have been made to allow shifting of the azimuth spectrum, which is advantageous for MTI, spotlight and large-squint processing.

The Inertial Measurement Unit (IMU) contains separate accelerometers and gyros that gather motion and attitude information, and includes Analogue/Digital Converters controlled by the Pulse Control module. Similar, the Pulse Control module reads the GPS receiver's serial data and synchronisation lines. The GPS antenna is placed outside the pod at a suitable aircraft location (e.g. top of the wing); only the GPS receiver of is placed inside the pod. IMU and GPS data acquisition is fully controlled by the Pulse Control module, enabling tight synchronisation between motion data and radar data. Processing of the motion and attitude data is done in software on the Radar Control host, which uses the GPS and IMU raw data to calculate the actual radar position and attitude, and sends this over the radar bus to the Data Chain for recording and off-line processing. The radar attitude is also used to calculate the antenna beam setting.

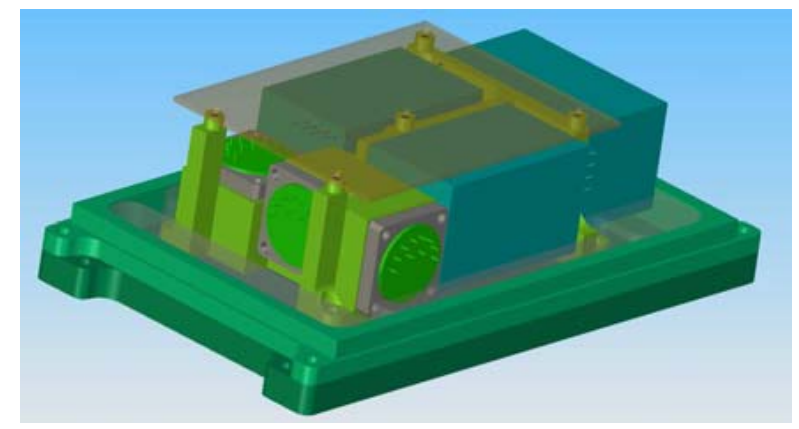

Figure 8 Mechanical set-up of the IMU 


\section{RADAR PERFORMANCE}

The radar his capable of SAR and MTI modes, in particular SAR strip and Spot modes, and MTI Wide-Area and Fast Sector Scan modes. Maritime MTI (maritime survaillance) is also foreseen, although the field of view is inevitable limited by the pod mounted design, so that forward MTI is not possible. The maximum Spot SAR resolution aims at $30 \mathrm{~cm}$ typical and $10 \mathrm{~cm}$ maximum. Range performance is obviously very dependent on the size of the radar. The small radar $(24 \times 4)$ will have a range performance of about $40 \mathrm{~km}$ is Strip SAR mode, and $35 \mathrm{~km}$ for MTI. The large version of the radar will have a typical range performance of $100 \mathrm{~km}$ and $90 \mathrm{~km}$ for Strip SAR and MTI modes respectively. Note that range performance is also a function of platform speed and will therefore vary according to the particular platform and associated operational conditions. It can be assumed however, that the smaller radar will be mounted on smaller and generally slower platforms.

Wide Area MTI modes will operate within a sector of up to +/- 60 degrees. The revisit times range from 30 to 60 seconds for these modes, while the FSS modes have a typical revisit of
6 seconds, I order to allow target tracking. The FSS area is about $6 \times 6 \mathrm{~km}^{2}$ for the smaller radar, $10 \times 10 \mathrm{~km}^{2}$ for the large radar. Good target localisation (CEP) and minimum detectable velocity properties are obtained using the 3 subapertures with Space Time Adaptive processing.

In maritime mti modes, the range of targets, i.e. radar cross sections is extremely wide, from small speed boats $\left(1-2 \mathrm{~m}^{2}\right)$, to large vessels $\left(>100 \mathrm{~m}^{2}\right)$. Therefore range performance can only be specified for certain target classes and varies from 25 to $60 \mathrm{~km}$ for the small radar, and $50 \mathrm{~km}$ to $135 \mathrm{~km}$ for the large radar.

\section{CONCLUSIONS}

The MiniSAR system and ART/VALKYR radar design offer very flexible solutions for a wide range of ground surveillance applications, and can be mounted on various platforms thanks to careful pod design and cooling solution, with minimum impact on the aircraft.

\section{REFERENCES}

[1] W.L. van Rossum, B.C.B. Vermeulen, SAR/MTI on small airborne platforms, Proceedings EUSAR 2006 